\title{
GRB Progenitors and Observational Criteria
}

\author{
Bing Zhang \\ Department of Physics and Astronomy, University of Nevada, Las Vegas, NV 89154, USA \\ email: zhang@physics.unlv.edu
}

\begin{abstract}
Phenomenologically, two classes of GRBs (long/soft vs. short/hard) are identified based on their $\gamma$-ray properties. The boundary between the two classes is vague. Multiwavelength observations lead to identification of two types of GRB progenitors: one related to massive stars (Type II), and another related to compact stars (Type I). Evidence suggests that the majority of long GRBs belong to Type II, while at least the majority of nearby short GRBs belong to Type I. Nonetheless, counter examples do exist. Both long-duration Type I and short-duration Type II GRBs have been observed. In this talk, I review the complications in GRB classification and efforts in diagnosing GRB progenitors based on multiple observational criteria. In particular, I raise the caution to readily accept that all short/hard GRBs detected by BATSE are due to compact star mergers. Finally, I propose to introduce "amplitude" as the third dimension (besides "duration" and "hardness") to quantify burst properties, and point out that the "tip-of-the-iceberg" effect may introduce confusion in defining the physical category of GRBs, especially for low-amplitude, high-redshift GRBs.
\end{abstract}

Keywords. gamma-ray bursts; classification

\section{Phenomenological vs. physical classification schemes}

Observations of GRBs with BATSE led to the identification of two phenomenological classes of GRBs in the duration-hardness $\left(T_{90}-\mathrm{HR}\right)$ plane: long/soft vs. short/hard (Kouveliotou et al. 1993). The boundary between the two classes is vague. The duration separation line is around 2 seconds in the BATSE band (30 keV - $2 \mathrm{MeV}$ ). Long and short GRBs roughly comprise $3 / 4$ and $1 / 4$ of the total population.

The main issue of applying the $T_{90}$ criterion to define the class of a GRB is that $T_{90}$ is detector dependent. GRB pulses are typically broader at lower energies. Also a more sensitive detector tends to detect weaker signals which would be otherwise buried in the noise. Indeed, observations carried out with softer detectors such as HETE-2 and Swift brought confusion to classifications. Among a total 476 GRBs detected by Swift BAT (sensitive in $15 \mathrm{keV}-150 \mathrm{keV}$ ) from Dec. 192004 to Dec. 212009 , only $8 \%$ have $T_{90}<2 \mathrm{~s}$ (Sakamoto et al. 2011), much less than the $\sim 1 / 4$ fraction of the BATSE sample. An additional $2 \%$ of Swift GRBs have a short/hard spike typically shorter than or around $2 \mathrm{~s}$, but with an extended emission lasting 10's to $~ 100$ seconds. These "short GRBs with E.E." (e.g. Norris \& Bonnell 2006) have $T_{90} \gg 2$ s as observed by Swift, but could be "short" GRBs if they were detected by BATSE. So the unfortunate consequence of the $T_{90}$ classification is that the membership in a certain category of the same GRB could change when the detector is changed. Nonetheless, the confusion in $T_{90}$ classification only arises in the "grey" area between the two classes. For most GRBs, one can still tell whether they are "long" or "short".

Follow-up afterglow and host galaxy observations of GRBs led to the identification of at least two broad categories of progenitors. Observations led by BeppoSAX, HETE-2, and Swift suggest that at least some long GRBs are associated with supernova Type Ic 
(e.g. Hjorth et al. 2003; Campana et al. 2006; Pian et al. 2006). Most long GRB host galaxies are found to be dwarf star-forming galaxies (Fruchter et al. 2006). These facts establish the connection between long GRBs and the death of massive stars (Woosley 1993). The breakthrough led by Swift unveiled that some nearby short GRBs (or short GRBs with E.E.) have host galaxies that are elliptical or early-type, with little star formation (Gehrels et al. 2005; Barthelmy et al. 2005; Berger et al. 2005). This points towards another type of progenitor that does not involve massive stars, but is likely related to compact stars, such as NS-NS or NS-BH mergers (e.g. Eichler et al. 1992).

The current observational data suggest that the majority of long GRBs belong to the massive star progenitor category (Type II), and at least the majority of nearby short GRBs belong to the compact star category (Type I; see e.g. Zhang 2006 for a discussion on physical classification scheme of GRBs). Nonetheless, the cozy picture of "long GRBs = Type II GRBs", and "short GRBs = Type I GRBs" does not always hold. Long duration Type I GRBs such as GRB 060614 have been observed (e.g. Gehrels et al. 2006; Zhang et al. 2007), which show deep upper limits on the brightness of any associated supernova, as well as a local galactic environment with low star formation rate (e.g. Gal-Yam et al. 2006). Some short-duration Type II GRBs are also observed, including three highestredshift, "rest-frame short" (i.e. $T_{90} /(1+z)<2$ s) GRBs, i.e. GRB 080913 at $z=$ 6.7 (Greiner et al. 2009), GRB 090423 at $z=8.2$ (Tanvir et al. 2009), and GRB 090429B at $z=9.4$ (Cucchiara et al. 2011), and one observer-frame short GRB 090426 at $z=2.609$ (Levesque et al. 2010; Xin et al. 2011).

It is then desirable to answer the following challenging question:

\section{How can one tell the physical class of a GRB based on the observational data?}

In order to address this question, let's remind ourselves what are the observational facts that made us believe in the existence of two distinct classes of progenitors. Zhang et al. (2009) summarized 12 multi-wavelength observational criteria that could be connected to the physical nature of a GRB, which include (1) supernova association; (2) specific star formation rate of the host galaxy; (3) position inside the host galaxy; (4) duration; (5) hardness; (6) spectral lag; (7) statistical correlations (e.g. $E_{p}-E_{\gamma, \text { iso }}, E_{p}-L_{\gamma, \text { iso }}$, $L$-lag); (8) energetics and beaming; (9) afterglow properties (medium density and spatial profile); (10) redshift distribution; (11) luminosity function; and (12) gravitational wave signal. Except criterion (12), which could be more definitive but is more difficult to carry out, other criteria have been applied to the known GRBs. Criteria (10) and (11) are statistical, relying on a large sample of data. Some interesting results have been obtained, which will be discussed in $\S 3$ below. Other criteria can be applied to individual GRBs, and the above particular order of the criteria is based on how closely relevant a particular criterion is to the progenitor. One can see that "duration" and "hardness", which are used in phenomenological classification, are not direct indications of GRB progenitor. This is not surprising, since the bimodal distribution has been known since the BATSE era, but it was after BeppoSAX, HETE-2, and Swift when people identified the two broad progenitor types. The first three criteria (SN, host galaxy, and position within the host galaxy) carry much more weight in defining the physical category of a GRB.

A flowchart to diagnose the physical category of a GRB based on multiple observational criteria was proposed in Zhang et al. (2009), see Fig. 1. Several noticeable features of the flowchart are the following: 1. Even though duration can be used to roughly separate GRBs, there are several bridges that allow bursts to break the duration separation line. 


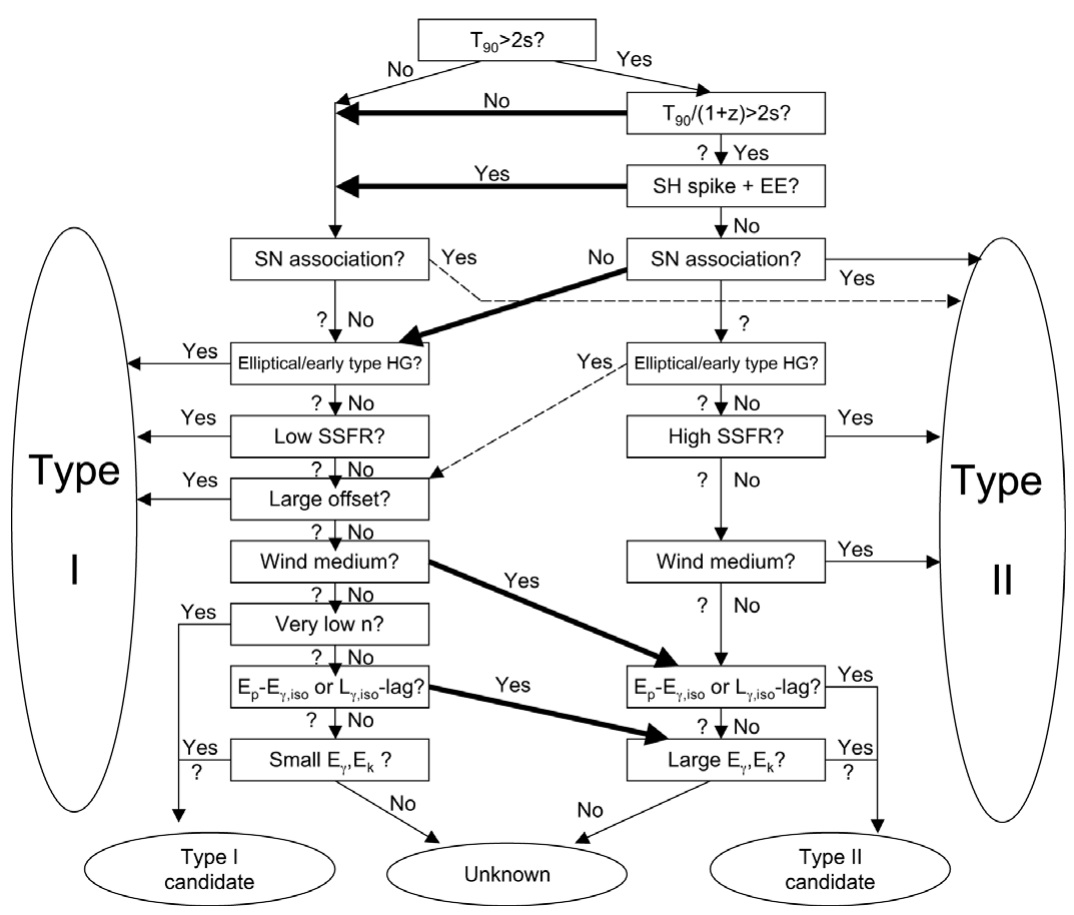

Figure 1. A flowchart using multiple observational criteria to diagnose the physical category of a GRB. After the initial duration criteria (which are used to start with a rough separation), the sequence of the applied criteria are based on their degree of relevance to the progenitor. From Zhang et al. (2009).

Noticeable examples include the long-duration Type I GRB 060614, rest-frame short high$z$ GRBs 080913, 090423, and 090429B, as well as observer-frame short GRB 090426. 2. The upper criteria (SNe and host galaxy properties) carry more weight, and can directly lead a burst to be Type I or Type II; 3. The lower criteria invoking afterglow modeling or empirical correlations carry less weight, and would only lead bursts to be Type I or Type II "candidates".

Such a flowchart has been applied to study currently observed long and short GRBs with afterglow data, and it proves to work well (Kann et al. 2010, 2011). In order to apply the flowchart, one needs to have a lot of extra information (afterglow, SN, host galaxy, redshift, etc.) other than prompt $\gamma$-ray properties. With $\gamma$-ray information only, one cannot determine the physical type of GRBs with high confidence. Nonetheless, one could give a "guess". This is particularly important for a GRB trigger team, since early on there is no afterglow, redshift, SN, and host information. An alert from the team would help the follow-up observers to decide how significant a burst is. The Swift team essentially applies the following flowchart to predicts the category of the GRB (Fig. 2).

Some other efforts have been carried out to use fewer parameters to identify the physical category of a GRB. For example, Lü et al. (2010) showed that for GRBs with $z$ measurements, the parameter $\varepsilon \equiv E_{\gamma, i s o, 52} / E_{p, z, 2}^{5 / 3}$ can be a good indicator. The high- $\varepsilon$ vs. low- $\varepsilon$ categories are found to be more closely related to Type II vs. Type I, respectively, rather than the traditional long vs. short classification. 


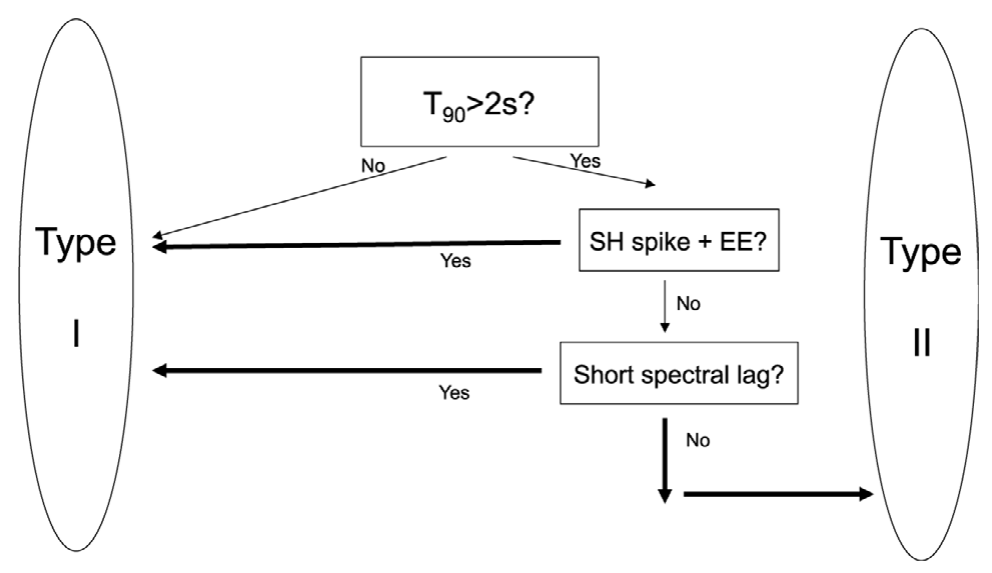

Figure 2. The flowchart used by the Swift team.

\section{Are all short/hard GRBs of a compact star merger origin?}

The leading model for Type I GRBs is NS-NS or NS-BH mergers. An immediate question would be: are all short/hard GRBs as detected by BATSE due to NS-NS or NS-BH mergers?

Most $z$-known short-duration GRBs are predominantly located at redshifts below 1 (Berger 2009). The peak luminosity spans in a wide range (Virgili et al. 2011), from $\sim 7 \times 10^{48} \mathrm{erg} \mathrm{s}^{-1}$ (for GRB 050509B, Gehrels et al. 2005) to $\sim 3.8 \times 10^{52} \mathrm{erg} \mathrm{s}^{-1}$ (for GRB 090510, De Pasquale et al. 2010). An intuitive response to the above question is "perhaps not". Unlike a massive star progenitor that has an extended envelope that can collimate the jet, a NS-NS merger system does not have a natural collimator. A broad jet can be launched, but never highly collimated (e.g. $\sim 30^{\circ}$, Rezzolla et al. 2011). The neutrino annihilation energy power is typically low. Even if the Blandford-Znajek mechanism is invoked, to produce a burst similar to GRB 090510 requires far-stretching of the models. Indeed, Zhang et al. (2009) suggested that high- $z$, high- $L$ short GRB may not be of a Type I origin. Panaitescu (2011) suggested a Type II origin of GRB 090510 based on its wind-like density profile in afterglow modeling.

There are two approaches to address whether all short GRBs are due to compact star mergers. The first approach is to use multiple criteria to identify a Gold sample of Type I GRBs, and check whether it is a good representation of the entire short/hard GRB population. Zhang et al. (2009) took this approach and concluded that it may not be. The second approach is to take the observed short GRBs as one population, and statistically compare it with the long GRB population to see the difference. The host galaxy study by Berger (2009) and Fong et al. (2010) is of this type. They show that short GRB host galaxies and the afterglow positions inside the hosts are indeed statistically different from those of long GRBs. The sample for such studies is still small, and is dominated by the nearby low- $L$ short GRBs (which are Type I). So the conclusion does not necessarily support that all BATSE short/hard GRBs are Type I.

In Virgili et al. (2011), we also performed an analysis using the second approach, but used the gamma-ray data (instead of the host galaxy data). We assume that the $z$-known short-duration GRBs detected by Swift and the $z$-unknown short/hard GRBs detected by BATSE belong to the same parent sample, and test whether the NS-NS and NS-BH merger models can simultaneously reproduce both daughter samples. As shown in Fig. 3, this is extremely difficult, if not impossible. The reason is that the $L-z$ distribution 

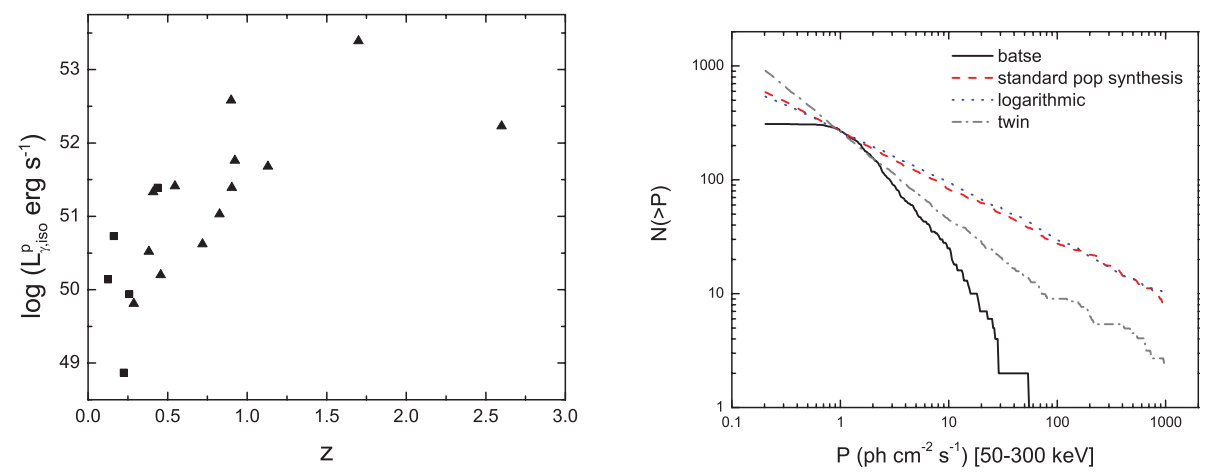

Figure 3. Left: the luminosity $(L)$ - redshift $(z)$ distribution of the $z$-known short GRB population detected by Swift (before June 2009). One can see low- $z$ clustering, and lack of significant increase of low- $L$ short GRBs with respect to high- $L$ short GRBs, suggesting a shallow luminosity function; Right: Within the compact star merger model, a shallow luminosity function is translated into a shallow peak flux distribution, which is inconsistent with the $\log N-\log P$ distribution of the BATSE short GRBs. This suggests that the compact star merger model cannot simultaneously reproduce both the $z$-known Swift sample and the $z$-unknown BATSE sample. From Virgili et al. (2011).

of the Swift sample demands a shallow luminosity function, since one does not see a significant increase of the number of low- $L$ short GRBs with respect to high- $L$ short GRBs. Since merger models predict a low- $z$ clustering due to the merger time delay with respect to star formation, this shallow luminosity function is translated to a shallow peak flux distribution $(\log N-\log P$ ), which cannot reproduce the BATSE short GRB $\log N-\log P$ data. Notice that even considering the highest fraction of "prompt mergers" (e.g. Belczynski et al. 2007), the shallow $\log N-\log P$ cannot be removed. There are two possibilities: 1. the Swift sample is not a good manifestation of the BATSE sample. A good fraction of the BATSE short GRBs are different, which may be a mix of Type I and Type II GRBs; 2. All short GRBs are Type I, but the NS-NS and NS-BH merger modes are not the correct one. Other models (e.g. accretion induced collapses) may be needed. In any case, using the short GRB sample to infer gravitational wave detection rate (e.g. Coward et al. 2012) may be pre-mature.

\section{Introducing "amplitude" as the third dimension and the confusion regimes of GRB classification}

In principle, a short GRB can be the "tip-of-the-iceberg" of a long GRB, if the extended longer duration emission is not bright enough to emerge from the detector background. So it is important to introduce the third dimension, i.e. "amplitude", besides "duration" and "hardness" to classify GRBs.

One can define a parameter $f \equiv F_{p} / F_{b}$, where $F_{p}$ is the peak flux, while $F_{b}$ is the background flux. For a long GRB, if we ideally scale down the flux so that the measured $T_{90}$ becomes shorter than 2 seconds, we will have a dimmer peak flux, $F_{p}^{\prime}$, for which one can define a new parameter $f_{\text {eff }} \equiv F_{p}^{\prime} / F_{b}$. For short GRBs, since $T_{90}<2$ s by definition, one has $f_{\text {eff }}=f$. For long GRBs, $f_{\text {eff }}$ defines a limit below which the "tip-of-the-iceberg" effect becomes important.

Such an exercise was carried out by Lü et al. (2012). Figure 4 shows the distribution of $f$ and $f_{\text {eff }}$ for long and short GRBs. The left panel shows both long and short GRBs can have high amplitude spikes. The right panel shows that if only the bright spike of 

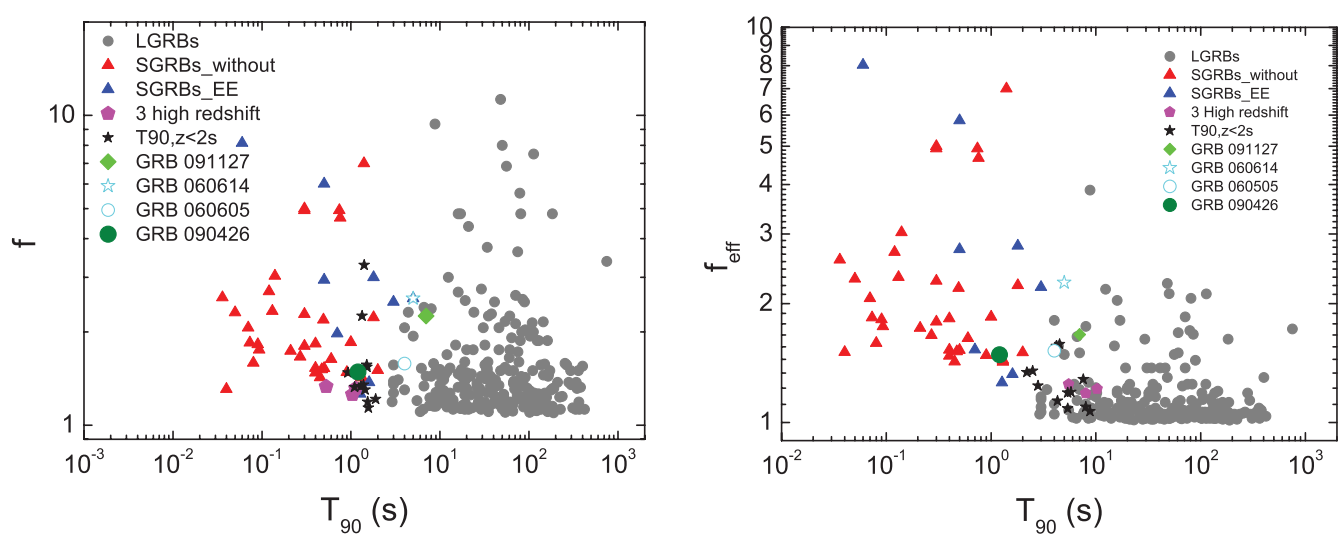

Figure 4. Left: The $T_{90}-f$ distribution of long and short GRBs in the Swift GRB sample. Both long and short GRBs can have high spikes with large $f$. Right: The $T_{90}-f_{\text {eff }}$ distribution of long and short GRBs. Short GRBs can have large $f$ 's suggesting that they are intrinsically short. However, some long GRBs can have $f_{\text {eff }}$ higher than a few. So short GRBs with low $f$ could be confused with "tip-of-the-iceberg" long GRBs. An example is GRB 090426, which has $f=1.48$. From H.-J. Lü et al., 2012, in preparation.

a long GRB is above the background, the typical $f_{\text {eff }}$ values are substantially dropped. Some short GRBs have a large enough $f$ not to be confused with the bright tip of long GRBs. This means that they are intrinsically short. However, some short GRBs have an $f$ comparable to $f_{\text {eff }}$ of some long GRBs, suggesting that they may be confused with a bright spike of long GRBs. It is interesting to note that GRB 090426 is a low $f$ short GRB $(f=1.48)$, which falls into the "confusion" area. Multiple observational criteria suggest that it is a Type II GRB (e.g. Levesque et al. 2010; Xin et al. 2011). So its short duration is likely due to the "tip-of-the-iceberg" effect.

One can also move a long GRB to progressively higher redshifts until its rest-frame duration is shorter than 2 seconds. Defining $f_{\mathrm{eff}, \mathrm{z}} \equiv F_{p, z}^{\prime} / F_{b}$, one can analyze the range of $f_{\text {eff }, \mathrm{z}}$ and compare with the $f$ values of the observed rest-frame short GRBs. It turns out that the three rest-frame short GRBs at high redshifts (GRBs 080913, 090423, 090429B) all have $f$ falling into the confusion region, suggesting that they are consistent with being the "tip-of-the-iceberg" of long-duration GRBs (Lü et al. 2012).

\section{Acknowledgments}

I thank my students/collaborators who carried out the heavy-duty tasks to tackle the complicated problems discussed in this talk, in particular, Hou-Jun Lü, Francisco Virgili, Bin-Bin Zhang, and En-Wei Liang. This work is supported by NSF through grant AST-0908362, and by NASA through grants NNX10AD48G, NNX10AP53G, and NNX11AQ08G.

\section{References}

Barthelmy, S. D. et al. 2005, Nature, 438, 994

Belczynski, K., Stanek, K. Z., \& Fryer, C. L. 2007, arXiv:0712.3309

Berger, E. et al. 2005, Nature, 438, 988

Berger, E. 2009, ApJ, 690, 231

Campana, S. et al. 2006, Nature, 442, 1008

Coward, D. et al. 2012, arXiv:1202.2179

Cucchiara, A. et al. 2011, ApJ, 736, 7 
De Pasquale, M. et al. 2010, ApJ, 709, L146

Eichler, D. et al. 1989, Nature, 340, 126

Fong, W., Berger, E., \& Fox, D. B. 2010, ApJ, 708, 9

Fruchter, A. S. et al. 2006, Nature, 441, 463

Gal-Yam, A. et al. 2006, Nature, 444, 1053

Gehrels, N. et al. 2005, Nature, 437, 851

Gehrels, N. et al. 2006, Nature, 444, 1044

Greiner, J. et al. 2009, ApJ, 693, 1610

Hjorth, J. et al. 2003, Nature, 423, 847

Kann, D. A. et al. 2010, ApJ, 720, 1513

Kann, D. A. et al. 2011, ApJ, 734, 96

Kouveliotou, C. et al. 1993, ApJ, 413, L101

Levesque, E. M. et al. 2010, MNRAS, 410, 963

Lü, H.-J., Liang, E.-W., Zhang, B.-B., \& Zhang, B. 2010, ApJ, 725, 1965

Lü, H.-J., Zhang, B., Liang, E.-W., \& Zhang, B.-B., 2012, in preparation

Norris, J. \& Bonnell, J. T. 2006, ApJ, 643, 266

Panaitescu, A. 2011, MNRAS, 414, 1379

Pian, E. et al. 2006, Nature, 442, 1011

Rezzolla, L. et al. 2011, ApJ, 732, L6

Sakamoto, T. et al. 2011, ApJS, 195, 2

Tanvir, N. et al. 2009, Nature, 461, 1254

Virgili, F., Zhang, B., O'Brien, P., \& Troja, E. 2011, ApJ, 727, 109

Woosley, S. E. 1993, ApJ, 405, 273

Xin, L.-P. et al. 2011, MNRAS, 410, 27

Zhang, B. 2006, Nature, 444, 1010

Zhang, B. et al. 2007, ApJ, 655, L25

Zhang, B. et al. 2009, ApJ, 703, 1696

\section{Discussion}

N. Tominaga: In your flowchart, a GRB is moved to Type I if it has no SN component. It is not necessary to have a bright $\mathrm{SN}$, because there is a diversity in SN brightness.

B. ZHANG: First, the flowchart is based on what we see not what we believe. Indeed some models can make faint SNe associated with GRBs. However, observationally there is no robust evidence yet for massive star GRBs with no SN association. GRB 060614 is a long GRB. However, it is not that different from the "smoking gun" "short" GRB 050724 . GRB 050724 is sitting near the edge of an early type galaxy. However, it is not that short, either. It has an early emission episode with $T_{90} \sim 3 \mathrm{~s}$, followed by an extended emission tail lasting longer than $100 \mathrm{~s}$ (Barthelmy et al. 2005). GRB 060614, on the other hand, has an early episode lasting for about $\sim 5 \mathrm{~s}$, followed by a softer oscillating tail of $\sim 100$ s (Gehrels et al. 2006). It would be essentially identical to GRB 050724 if it were 8 times less energetic (Zhang et al. 2007). Its host galaxy does not have active star formation, and the location of the burst does not track the bright star forming region in the host galaxy. All these suggest that GRB 060614 is not different from GRB 050724, and should be a Type I GRB. Interpreting it as a SN-less Type II GRB is based on the belief that all long GRBs are associated with massive stars. Otherwise, most nearby short GRBs (including the actually long-duration "short" GRB 050724) do not have SN associations, either. So why not interpreting them as SN-less core collapses as well? Second, in my flowchart, a long GRB without a SN association is not directly linked to Type I. It is only moved to the left hand side (which becomes a Type I candidate). One needs to go through other criteria to make the final judgment. GRB 060614 is eventually grouped to Type I, but this is because its host galaxy and location within the host galaxy also satisfy 
the criteria of a Type I GRB. If, in the future, one detects a long duration, SN-less GRB sitting in the bright region of an active star-forming galaxy, having large energetics, and satisfying the empirical correlations of other Type II GRBs, one would move it back to the right hand side of my flowchart, and it will be identified as a Type II candidate. If this indeed happens, then theoretical modeling of GRBs with very faint SNe becomes relevant.

F. Mirabel: Can you exclude that Doppler boosting dependence on viewing angle does not play any role in GRB classification? Namely, on duration and hardness?

B. Zhang: The Doppler effect plays an important role in the AGN field, but less in the GRB field. This is mostly because GRBs have higher Lorentz factors $(\Gamma>100)$ and wider jet opening angles $\left(\theta_{j} \sim 5^{\circ}\right)$ than AGNs, so that usually $\Gamma^{-1} \ll \theta_{j}$ is satisfied. For most geometry, the line of sight is within the jet cone. Observationally, long-duration GRBs typically have multiple peaks, with minimum variability of order milliseconds. So the long duration is the intrinsic property of the central engine, not due to Doppler broadening. Otherwise one should not see much smaller variability time scales. Also early optical afterglows of most GRBs show a decay behavior. This is consistent with the prediction of the on-beam geometry. If the line of sight is outside of the jet cone initially, one would expect to see a rising (or very shallow decay) lightcurve instead. Of course, there is a category of low-luminosity, long-duration GRBs that show one broad peak in the lightcurve. These bursts could be related to bursts viewed at a large off-beam angle with a low Doppler factor. A competitive model is that these are intrinsically different events, probably related to jets that barely breakout from the star, in contrast with highluminosity long GRBs that have successful jets. The two scenarios can be in principle differentiated with late radio afterglow data. The current data do not support the offbeam jet model. So, to answer your question, even though the viewing angle effect is not fully excluded, one can say that it plays a minor, if any, role in GRB classification.

E. NAKAR: I have a few comments. First, the separation line of $T_{90}=2 \mathrm{~s}$ is for BATSE. For other detectors (e.g. Swift), the separation line can be different. Second, with $\gamma$ ray information only, it is impossible to tell which physical category a GRB belongs to. Besides contamination of Type II in short GRBs, there could be contamination of Type I in long GRBs, too.

B. ZHANG: I fully agree with both comments. Regarding $T_{90}$, I agree that the 2 -second separation line is for BATSE only. The Swift team currently also uses 2-second as the separation. On the other hand, as is described in the flowchart (Fig. 1), the initial separation is not fundamental. One really needs to go through all the criteria before the final identification of the physical category of a burst is made. There are bridges to connect the two sides. So even if there was inaccuracy in the initial duration criterion, after going through the flowchart, a burst would land in the right physical category. Second, indeed there could be more Type I contaminations in the long duration GRBs. For example, if a long GRB (no short hard spike and no deep SN upper limit) sit in the outskirt of an early type galaxy, one has to be cautious to define its category. In the current flowchart, if this burst also had low energetics, it would be grouped to the "unknown" category. This is a field full of surprises. The flowchart was designed to our best knowledge in 2009, and it still works reasonably well until now (see e.g. Kann et al. 2010, 2011). However, it is possible that future observations may suggest that more "bridges" are needed in the flowchart, or even that the global structure of the flowchart has to be modified. 